\title{
Ovulation Induction and Intrauterine Insemination for Hypogonadotropic Hypogonadism
}

\author{
Siwatch S*, Suri V, Dhaliwal LK and Gainder S \\ Department of Obstetrics and Gynaecology, Post \\ Graduate Institute of Medical Education and Research, \\ India \\ *Corresponding author: Sujata Siwatch, Department \\ of Obstetrics and Gynaecology, Post Graduate Institute of \\ Medical Education and Research, Sector 12, Chandigarh, \\ India
}

Received: April 25, 2021; Accepted: May 15, 2021; Published: May 22, 2021

\begin{abstract}
Hypogonadotropic Hypogonadism $(\mathrm{HH})$ is a rare gynae- endocrinological cause of anovulatory infertility. Gonadotropins are given to induce ovulation. Various ART techniques have been used for assisting conception in these women. In this study, we aimed to calculate the chances of success of ovulation and pregnancy rates after ovulation induction and intrauterine seminal insemination in $\mathrm{HH}$ women. We reviewed and analyzed the chances of success of ovulation and conception with ovulation induction and intrauterine seminal insemination in thirty two couples who underwent 56 intrauterine insemination cycles. The average age of these women was 27.2+/- 3.2 years. Ovulation was documented in 55/56 cycles. An average of $12.89+/-5.05$ days of stimulation was required to induce ovulation. The pregnancy rate was 50\%. Thus, intrauterine insemination offers a cheaper yet good alternate option of reproductive techniques in addition to ovulation induction with gonadotropins, in hypogonadotropic hypogonadism, especially in the low resource settings.
\end{abstract}

Keywords: Hypogonadotropic hypogonadism; Infertility; Female; Reproductive techniques; Assisted/economics

\section{Abbreviations}

HH: Hypogonadotropic Hypogonadism; BMI: Basal Metabolic Rate; FSH: FSH: Follicle Stimulating Hormone; LH: Leutinizing Hormone; HMG: Human Menopausal Gonadotropins; IUI: Intrauterine Insemination; ART: Assisted Reproductive Techniques

\section{Introduction}

Hypogonadotropic Hypogonadism $(\mathrm{HH})$ is an endocrinological condition which constitutes a small but definite group of anovulatory infertility patients. Due to hyposecretion of gonadotrophin releasing hormones in the hypothalamus or due to pituitary dysfunction, gonadal activity is limited and patients present with amenorrhea and infertility. Use of exogenous gonadotropin therapy and artificial reproductive techniques for infertility management is well documented in these patients [1].

Though the results of treatment with in-vitro fertilization are good, the cost of procedure is a limitation. However, there is paucity of literature on the use and results of intrauterine insemination in these women.

The objectives of this study were to establish 1 . The prevalence of hypogonadotropic hypogonadism in infertile patients presenting to the Infertility clinic in our institute, 2 . the chances of success of ovulation with gonadtropins, 3. pregnancy rates after ovulation in $\mathrm{HH}$ women, and 4. to study the relation of BMI and the dose of gonadotropins required.

\section{Materials and Methods}

We reviewed the case records of women with anovulatory infertility with hypogonadotropic hypogonadism who presented to the infertility Clinic of department of Obstetrics and Gynecology in a tertiary care hospital from 2004-2013. Hypogonadotropic hypogonadism is characterized with amenorrhea, low levels of gonadotrophins (less than $5 \mathrm{IU} / \mathrm{ml}$ ) and absence of withdrawal bleeding following progesterone challenge.

As per the protocol in our clinic, women with anovulation underwent evaluation for hypothyroidism and hyperprolactinemia. The patients were subjected to a progesterone withdrawal test. Women with no withdrawal bleeding with progesterone then underwent evaluation of FSH and $\mathrm{LH}$ and were subjected to an estrogenprogesterone test. Women with $\mathrm{HH}$ were diagnosed with 1 . low levels of FSH and LH and 2. a positive estrogen-progesterone challenge. After evaluation of partner's semen, couples were counseled regarding the cause of anovulation and need for therapy with gonadotropins for the same. For stimulating ovulation, incremental doses of gonadotropins (HMG) were administered to these women and the response was assessed using transvaginal ultrasound. The starting dose was $150 \mathrm{IU}$ per day which was increased sequentially by 75 IU per week upto 450 IU, according to the ovarian response. Luteal phase support is given with progesterone and 2000IU biweekly hCG injections. IUI was done after 30 -36 hours of hCG trigger using 10,000IU hCG. The aim of ovulation induction was to develop one to two dominant follicles for ovulation and pregnancy. To increase the chances of conception in these ovulatory cycles, intrauterine insemination was planned and done after discussion with the couples.

In this study, we collected the demographic information, height, weight, BMI, baseline hormonal assays, gonadotropin dose administered, success of ovulation in gonadotropin cycles and pregnancy rates of $\mathrm{HH}$ women undergoing ovulation and IUI at the infertility clinic. The data was de-identified and analyzed for outcomes of success of ovulation and pregnancy. We also studied the probable relation of the dose of gonadotropin required for ovulation 
with the body mass index.

\section{Results}

A total of 38 women presenting to the infertility clinic of our institute were diagnosed $\mathrm{HH}(0.62 \%)$. Thirty seven women had primary amenorrhea while one had secondary amenorrhea after surgical excision of non functional pituitary microadenoma. The majority of these women had received multiple cycles of combined pills for amenorrhea previously. One couple also had male factor infertility due to asthenospermia.

Of these 38 women, 32 underwent ovulation induction with gonadotropins (HMG) followed by intrauterine insemination. The average age of these women was 27.16+/-3.2 years (range 22-33 years). Average age of first withdrawl bleed was $17.9+/-3.3$ years, achieved after sequential/ combined intake of estrogen-progesterone pills. The mean duration of infertility was $3.6+/-2.9$ years. The FSH and LH levels were $1.3+/-0.95$ and $0.88+/-0.87$ IU respectively. The demographic details, cycle characteristics and outcomes are detailed in Table 1 .

Thirty two couples underwent 56 intrauterine insemination cycles. Ovulation was documented in all but one cycle. Intrauterine insemination was done for all ovulatory cycles. An average of $12.89+/$ 5.05 days of stimulation was required to induce ovulation. The starting dose was $150 \mathrm{IU}$ per day which was increased sequentially by 75 IU per week upto 450 IU. The average dose of gonadotropins used in each cycle was $2171.80+/-1109$ IU or $28.95+/-14.79$ ampoules. The dose correlated with the BMI of the patients. The pregnancy rate after intrauterine insemination was 50\% (16 of 32 cases), of which 9 women conceived after the first cycle, 6 after the second and 1 after the third cycle.

\section{Discussion}

$\mathrm{HH}$ is a rare cause of female infertility [2]. It is thus difficult to recruit a large number of patients with a rare condition such as hypogonadotropic anovulation [3]. HH patients present with delayed menarche, amenorrhea, low levels of gonadotrophins and absence of withdrawal bleeding following progesterone challenge [4]. Primary hypogonadotropic hypogonadism is congenital whereas acquired causes of HH may include tumors, infiltrative diseases, infections and trauma. Idiopathic hypogonadotropic hypogonadism or Kallmans syndrome constitutes 1: 50,000 of infertile patients [5] and is notable for absence of pituitary tumor and is associated with other congenital abnormalities(cleft palate, renal agenesis, cardiac abnormality, abnormal platelet function) [4]. 27-45 percent of these women are anosmic or hyposmic [6,7]. In our study, none of the patients complained of hyposmia or anosmia though no formal evaluation of the same was done.

Gonadotrophins, with or without use of gnRH agonists and growth hormones have been used to induce follicle growth and maturation, to treat infertility [8]. It is also speculated that treatment of $\mathrm{HH}$ women with few cycles of combined pills builds up the endometrium and reduces the dose of gonadotrophins required for stimulation [9]. In our study too, most women received two to three cycles of combined pills before the cycle for ovulation and IUI.

Previous studies on ART including $\mathrm{HH}$ patients have shown that both the treatment duration and gonadotropin consumption were higher compared with patients with other aetiologies of infertility $[3,10]$. Even very high doses of gonadotrophins used in these patients have not been shown to be toxic to oocytes and embryos [1]. Due to low levels of FSH, LH and estrogen and small ovaries, these usual predictors of ovarian response to stimulation are not used in these patients and only the response to previous cycles can help us predict response pattern [10]. All the same, polycystic ovaries as compared to small ovaries have been noted to have a better response to stimulation [11]. In our study, $4 / 32$ (12.5\%) patients were found to have polycystic ovaries. In our study, the doses of gonadotrophins required correlate to the weight and BMI. It is well known that abdominal obesity reduces fertility in both sexes. The altered/disturbed levels of inhibin, SHBG and related changes lead to a condition of relative hyperandrogenism in obese women and of hypotestosteronemia and even true hypogonadotropic hypogonadism in obese men [12].

$\mathrm{HH}$ women are good responders to ART, even in aged patients $[1,13]$. Such aged $\mathrm{HH}$ patients require higher gondotrophin doses, the pregnancy rate is about $50 \%$ [1]. Yokoi et al compared various treatment regimens of stimulation in $\mathrm{HH}$ women and reported a higher rate of multiple pregnancy, number of growing follicles, conception and ovarian hyperstimulation rates with the conventional protocol of $150 \mathrm{IU}$ hMG daily [14]. At our institute, we used a protocol of step up of gonadotrophins according to the response assessed on ultrasound.

Most studies on infertility therapy for hypogonadotropic hypogonadism women have studied the use of ART $[10,11,15]$. In fact, treatment with gonadotrophins followed by IUI has been questioned for its cost effectiveness [16]. The 50\% pregnancy rate with gonadotrophins and IUI in our experience is comparable to the pregnancy rates in previous studies that used ART. In fact, the lower cost of procedure of IUI versus IVF makes it a more cost effective option.

Fifty percent of the women conceived with intrauterine insemination in our study. The multiple pregnancy rates were $4 / 16$ (25\%). These women received 2000IU of human chorionic gonadotrophin biweekly usually till about 12 weeks and in some for 16-18 weeks of pregnancy to support the pregnancy till placental function develops. The abortion rate was $3 / 16(18.7 \%)$ of those who followed up at our institute for antenatal check up. Ulug et al reported a multiple pregnancy rate of $13 \%$ and pregnancy loss rate of $8 \%$ in $58 \mathrm{HH}$ women who received ART treatment [15]. The BMI of these patients was $21.09 \pm 1.3$. Tadokoro et al have reported a pregnancy loss rate of $27 \%$ [17]. Huseyin et al also reported a pregnancy rate of $12.7 \%$ and $28.3 \%$ per cycle and per patient, respectively [5].

Our study is limited by the small number of patients and retrospective design. Future studies on larger number of patients may shed more light on the correlation of dose of gonadotropins needed for inducing ovulation with the weight and BMI of these patients.

To conclude, hypogonadotropic hypogonadism infertility patients have a good success rate of ovulation with gonadotropin stimulation. Intrauterine insemination offers a cheaper yet good alternate option of reproductive techniques in addition to ovulation induction with gonadotrophins, especially in the low resource settings. 


\section{Acknowledgement}

Dr Kamla Rani Gupta \& Dr Smitu Sachdev for their contribution in data collection and management of the patients.

\section{Impact Statement}

What is already known on this subject? infertility in Hypogonadotropic hypogonadism is responsible for both males and females. Female infertility is primarily due to anovulation. Gonadotropins are used to stimulate ovulation in these women, which is both expensive and time consuming. Assisted reproductive techniques are commonly used alongside to improve the chances of conception.

What the results of this study add?

The results of the study reinforce that infertility from hypogonadotropic hypogonadism in women can be successfully treated with intrauterine insemination.

What the implications are of these findings for clinical practice and/or further research?

Most studies on management of female infertility with hypogonadotropic hypogonadism report the high success of use of in-vitro fertilization. However, the more economical procedure of controlled ovarian stimulation and intrauterine insemination has both good success and is more cost effective for treating female factor infertility.

\section{References}

1. Kumbak B, Kahraman S. Women with hypogonadotropic hypogonadism cycle characteristics and results of assisted reproductive techniques. Acta Obstet Gynecol Scand. 2006; 85: 1453-1457.

2. Fraietta R, Zylberstejn DS, Esteves SC. Hypogonadotropic hypogonadism revisited. Clinics (Sao Paulo). 2013; 68: 81-88.

3. Burgués S. The effectiveness and safety of recombinant human LH to support follicular development induced by recombinant human FSH in WHO group I anovulation: evidence from a multicentre study in Spain. Hum Reprod. 2001; 16: $2525-2532$

4. Battaglia C, Salvatori M, Regnani G, Giulini S, Primavera MR, Volpe A Successful induction of ovulation using highly purified follicle-stimulating hormone in a woman with Kallmann's syndrome. Fertil Steril. 2000; 73: 284286.

5. Huseyin K, Berk B, Tolga K, Eser O, Ali G, Murat A. Management of ovulation induction and intrauterine insemination in infertile patients with hypogonadotropic hypogonadism. J Gynecol Obstet Hum Reprod. 2019; 48: 833-838.

6. Bhagavath B, Ozata M, Ozdemir IC, Bolu E, Bick DP, Sherins RJ, et al. The prevalence of gonadotropin-releasing hormone receptor mutations in a large cohort of patients with hypogonadotropic hypogonadism. Fertil Steril. 2005; 84: 951-957.

7. Bhagavath B, Podolsky RH, Ozata M, Bolu E, Bick DP, Kulharya A, et al Clinical and molecular characterization of a large sample of patients with hypogonadotropic hypogonadism. Fertil Steril. 2006; 85: 706-713.

8. Hayashi M, Tomobe K, Hoshimoto K, Ohkura T. Successful pregnancy following gonadotropin therapy in a patient with hypogonadotropic hypogonadism resulting from craniopharyngioma. Int J Clin Pract. 2002; 56: 149-151.

9. Dokuzeylul N, Kahraman S, Karlikaya G, Karagozoglu H, Kavrut M, Ersahin A. Clinical Outcome of Women $\leq 37$ years with Hypogonadotropic Hypogonadism Undergoing Intracytoplasmic Sperm Injection and in-vitro Fertilization. Fertil Steril. 2010; 94: S250.

10. Lewit N, Kol S. The low responder female IVF patient with hypogonadotropic hypogonadism: do not give up! Fertil Steril. 2000; 74: 401-402.

11. Shoham Z, Conway GS, Patel A, Jacobs HS. Polycystic ovaries in patients with hypogonadotropic hypogonadism: similarity of ovarian response to gonadotropin stimulation in patients with polycystic ovarian syndrome. Fertil Steril. 1992; 58: 37-45.

12. Pasquali R. Obesity, fat distribution and infertility. Maturitas. 2006; 54: 363 371.

13. Ghaffari F, Arabipoor A, Lankarani NB, Etminan Z, Tehraninejad ES. Assisted reproductive technique outcomes in hypogonadotropic hypogonadism women. Ann Saudi Med. 2013; 33: 235-240.

14. Yokoi N, Uemura T, Murase M, Kondoh $Y$, Ishikawa M, Hirahara F. A modified hMG-GnRH method for the induction of ovulation in infertile women with severe hypogonadotropic amenorrhea. Endocr J. 2002; 49: 159-164.

15. Ulug U, Ben-Shlomo I, Tosun S, Erden HF, Akman MA, Bahceci M. The reproductive performance of women with hypogonadotropic hypogonadism in an in vitro fertilization and embryo transfer program. J Assist Reprod Genet. 2005; 22: 167-171

16. Dragojević S, Pazin V, Rakić S, Nikolić B, Jovanović T. Pregnancy using gonadotropins and performing intrauterine insemination in a woman with hypogonadotropic hypogonadism. Am J Reprod Immunol. 2006; 55: 164-167.

17. Tadokoro N, Vollenhoven B, Clark S, Baker G, Kovacs G, Burger H, Healy D. Cumulative pregnancy rates in couples with anovulatory infertility compared with unexplained infertility in an ovulation induction programme. Hum Reprod. 1997; 12: 1939-1944. 